\title{
Pneumothorax following combination chemotherapy with bevacizumab: A case report and review of the literature
}

\author{
TAHA ALRIFAI $^{1}$, RAYA SABA ${ }^{2}$, DANA RIFAI ${ }^{3}$, SARBAGYA PANDIT $^{1}$ and KELLEY E. KOZMA ${ }^{4}$ \\ ${ }^{1}$ Department of Internal Medicine, Saint Joseph Hospital, Chicago, IL 60657; ${ }^{2}$ Department of Hospital Medicine, \\ John T. Milliken Department of Medicine, Washington University, St. Louis, MO 63110; \\ ${ }^{3}$ Department of Internal Medicine, St. Catherine Hospital, East Chicago, IN 46312; \\ ${ }^{4}$ Department of Hematology and Oncology, Saint Joseph Hospital, Chicago, IL 60657, USA
}

Received November 28, 2018; Accepted May 16, 2019

DOI: $10.3892 / \mathrm{mco} .2019 .1868$

\begin{abstract}
Bevacizumab (BV) is a humanized monoclonal antibody that inhibits angiogenesis by targeting vascular endothelial growth factor (VEGF). The addition of BV to combination chemotherapy has been shown to improve the outcomes in several malignancies, including colorectal carcinoma (CRC). However, the use of BV has been associated with adverse effects, including hypertension, hemorrhage, proteinuria, delayed wound healing and bowel perforation. Pneumothorax (PTX) as an adverse event associated with BV use has rarely been reported. We herein report the case of a 68 -year-old female patient with a history of metastatic CRC treated with combination chemotherapy, including BV, who presented with complaints of shortness of breath and was found to have a right-sided PTX.
\end{abstract}

\section{Introduction}

The vascular endothelial growth factor (VEGF)/VEGFreceptor pathway plays a key role in malignant tumor angiogenesis (1). This has led to the development of humanized monoclonal antibodies targeted against this pathway to inhibit tumor angiogenesis. Bevacizumab (BV) was the first approved anti-VEGF antibody (2) and it has become a standard part of several combination chemotherapy regimens used in patients with metastatic colorectal cancer (mCRC), after having been shown by several clinical trial to exert a statistically significant favorable effect on overall survival (OS) and progression-free survival (PFS) (3). Some of the most common adverse events associated with the use of BV include hypertension, hemorrhage, proteinuria, delayed wound healing and bowel perforation (4). Some have reported increased risk of arterial

Correspondence to: Dr Taha Alrifai, Department of Internal Medicine, Saint Joseph Hospital, 2900 North Lake Shore Drive, Chicago, IL 60657, USA

E-mail: tahaalrifai@gmail.com

Key words: bevacizumab, vascular endothelial growth factor inhibitors, oncology, pneumothorax, adverse reactions events, mainly cardiovascular and cerebrovascular events (5). However, pneumothorax (PTX) has rarely been reported in association with the use of BV (6-12). We herein report the case of a 68-year-old female patient who was undergoing third-line chemotherapy with folinic acid, fluorouracil (5-FU) and oxaliplatin (FOLFOX) plus BV when she presented with shortness of breath (SOB) and was diagnosed with pneumohydrothorax. We consider the present case to be important, as it sheds light on a rare adverse event associated with the use of $\mathrm{BV}$, in an era where VEGF-inhibitors are being implemented in the treatment of several malignancies. Clinicians should be aware of this potential adverse event in order to possess a high clinical index of suspicion for making this diagnosis.

\section{Case report}

A 68-year-old female patient presented in January 2018 to Saint Joseph's Hospital (Chicago, USA) with complaints of worsening SOB for 1 month, with an associated right-sided pleuritic chest pain and a dry cough. On physical examination, the patient was found to be mildly tachycardic to 102 beats per min, she was not tachypneic and her oxygen saturation was 98\% breathing ambient air. Physical examination was notable for decreased breath sounds over the right lower lung fields. Otherwise, the physical examination was unremarkable. This presentation was 1 week after the completion of the 4th cycle of combination chemotherapy with FOLFOX and BV for mCRC.

The patient was initially diagnosed with stage IIIB mCRC 9 years earlier, for which she had undergone sigmoidectomy and completed 12 cycles of adjuvant chemotherapy with FOLFOX. Four years later, a positron emission tomography with computed tomography (PET/CT) scan revealed disease recurrence, with 3 metastatic lung lesions. The patient subsequently underwent Cyberknife therapy followed by 12 cycles of leucovorin, 5-FU and irinotecan (FOLFIRI) with cetuximab, followed by maintenance therapy with cetuximab. Two years later, a CT scan of the abdomen revealed evidence of a new left adrenal mass, which was treated with radiofrequency ablation; a biopsy was consistent with metastatic adenocarcinoma. The patient was then placed on combination chemotherapy with FOLFIRI and BV followed by maintenance capecitabine, which she tolerated well, and a PET/CT scan 1 year later showed 


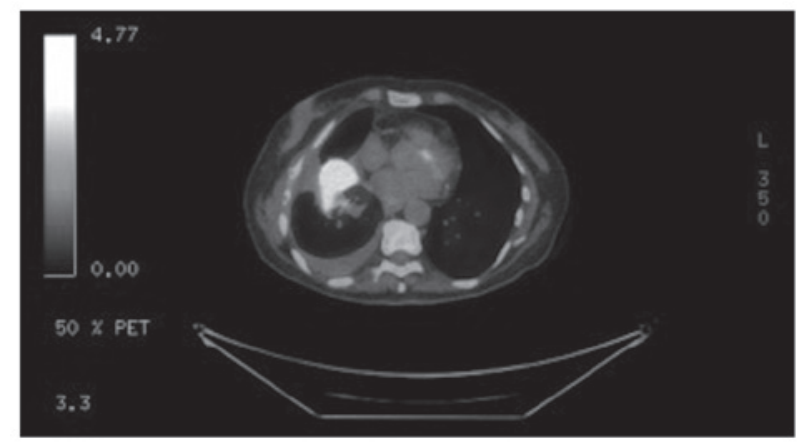

Figure 1. Positron emission tomography-computed tomography scan showing increased metabolic activity within a right lower lobe lung mass.

no evidence of disease. However, 3 months later, an elevation in her carcinoembryonic antigen (CEA) levels was observed, and a PET/CT scan (Fig. 1) revealed disease recurrence with lesions in the right lung base, subcarinal mediastinal lymph node and a left adrenal mass. The patient was then started on combination chemotherapy with FOLFIRI and the endothelial growth factor receptor inhibitor panitumumab. Disease progression was identified on restaging CT scan, along with an increase of the CEA levels; hence, the decision was made to start the patient on FOLFOX and BV. The patient had a family history of prostate cancer in her father and heart failure in her mother. The patient was a never smoker, and she did not drink alcohol or use illicit drugs.

The patient underwent a chest X-ray (Fig. 2), which revealed evidence of a moderate hydropneumothorax encompassing $40 \%$ of the right hemithorax volume. There was also a stable opacity within the right perihilar region, which correlated with known metastatic disease.

The patient's PTX was considered to be secondary to the presence of an underlying pulmonary metastatic lesion in the setting of BV use. The patient was a never smoker, with no underlying obstructive lung disease; she had no chronic pulmonary conditions other than her metastatic disease that had been present for several years; she had also not had any recent chest trauma, or undergone any recent procedures.

The patient underwent ultrasound-guided placement of a 10 French chest tube with evacuation of $200 \mathrm{ml}$ of air. The chest tube was kept on water seal for 5 days, then clamped the next day, and a repeat X-ray revealed resolution of the PTX (Fig. 3). The chest tube was removed, and the patient was discharged.

BV was indefinitely discontinued and substituted with cetuximab. After the 7th cycle of chemotherapy, a restaging PET/CT scan revealed enlargement of the right basilar lung mass with new right hilar and mediastinal lymphadenopathy, indicating progression of disease (POD). The patient completed 12 cycles of the current chemotherapy regimen, and POD occurred again, after which time she was started on trifluridine and tipiracil. The patient was then admitted to the hospital with acute encephalopathy and was found to have sepsis secondary to pneumonia. A CT scan of the head revealed a $0.9-\mathrm{cm}$ metastatic lesion in the left frontal lobe. Palliative and hospice care were recommended with services initiated during the same hospitalization. The patient succumbed to the disease 1 week after admission.

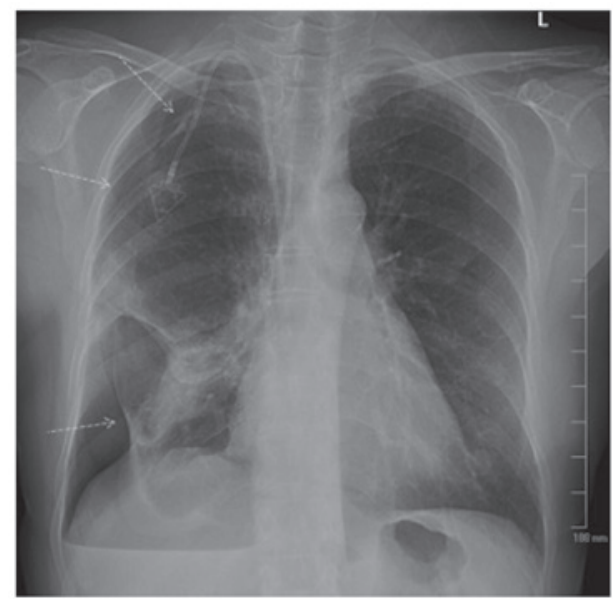

Figure 2. Right-sided pneumothorax with pleural effusion, encompassing $\sim 40 \%$ of the right hemithorax (arrows indicate the visceral pleura line).

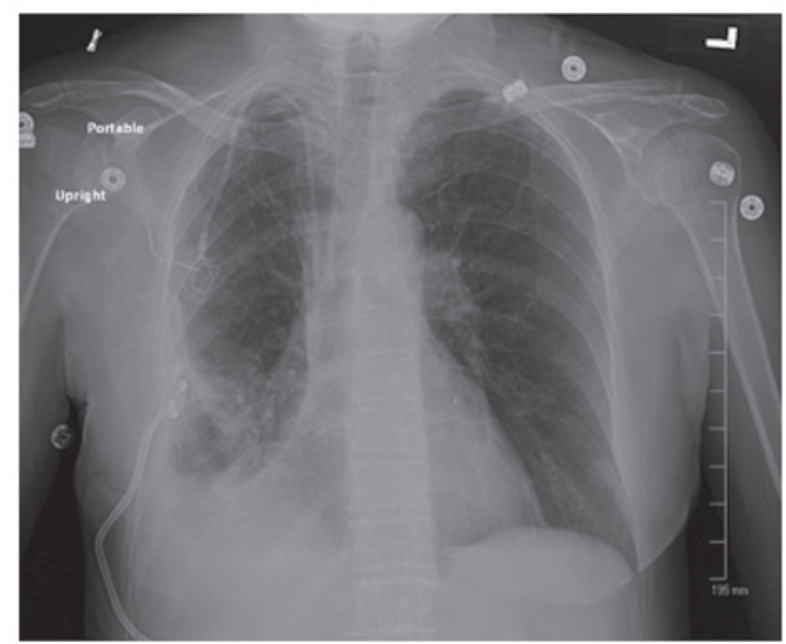

Figure 3. Repeat chest X-ray showing resolution of previously noted pneumothorax with chest tube placement.

\section{Discussion}

$\mathrm{BV}$ is a recombinant humanized monoclonal antibody that exerts antitumor effects by binding to VEGF and inhibiting tumor angiogenesis (1). BV has been widely used in the treatment of solid tumors, including CRC, renal cell carcinoma, ovarian carcinoma and several others (4). BV has several side effects pertaining to its antiangiogenic effects. However, PTX as a complication of BV use has rarely been reported. MEDLINE was searched using two keywords, namely 'BV' and 'pneumothorax'; the search yielded a total of 15 results, 7 of which were case reports relevant to the topic, and 6 of which had text available in English (Table I).

In 5 of the reviewed cases, including the present case, pulmonary metastases were present. However, Zhang et al reported a case of PTX following treatment of fibrosarcoma where no lung lesions were present. Hence, PTX may occur even in the absence of metastatic disease. Patients with mCRC were more likely to develop PTX with BV $(n=3)$, as compared with fibrosarcoma $(n=1)$, synovial sarcoma $(n=1)$ and breast cancer $(n=1)$. It remains unclear whether this observation is a 
matter of chance, considering that $\mathrm{mCRC}$ is the second most common malignancy to metastasize to the lung (13), and the fact that $\mathrm{mCRC}$ is one of the most common indications for BV treatment. PTX in association with BV was observed following a variable number of therapy cycles.

Srinivas and Varadhachary proposed that malignancy-associated PTX may result from tumor compression of the bronchial wall, leading to the formation of a one-way valve, resulting in air trapping and eventual rupture. Another potential cause is bronchopleural fistula formation as a result of effective chemotherapy, and spontaneous vascular occlusion within the tumor itself (14). Hence, it is possible that the antiangiogenic effect of $\mathrm{BV}$, which leads to distortion of the tumor vasculature, may lead to PTX in peripherally located tumors. In addition to the suggested tumor-related mechanisms of PTX development, a study by Kasahara $e t$ al in animal models found that chronic treatment with VEGF inhibitors led to the distortion of the alveolar structure through the induction of cell apoptosis, suggesting that this may contribute to the development of emphysema (15), which is a risk factor for PTX.

Our patient had also undergone radiosurgery with Cyberknife 4 years prior to her current presentation. PTX has been reported with Cyberknife therapy following CT-guided fiducial placement (16), an acute complication that was not observed in our patient. CT scan of the chest following Cyberknife therapy did not show evidence of lung damage that would be attributable to that therapy. However, it is possible that our patient developed lung parenchymal damage secondary to radiation that was undetectable on imaging modalities and may have contributed to the development of PTX. However, we consider the PTX that she developed to be associated with her most recent treatment with BV in the setting of lung metastatic disease, as the PTX developed after the 4th dose of BV, and the fact that this patient had also received several cycles of chemotherapy following Cyberknife therapy (12 cycles of FOLFIRI and cetuximab, and 24 cycles of FOLFIRI and panitumumab), which were well-tolerated. Our patient had received BV in the past, which she also tolerated well; however, it is worth noting that, at the time, the patient did not display evidence of lung metastatic disease on imaging. Therefore, underlying lung parenchymal disease, including lung metastatic disease, may place patients at an increased risk of developing BV-associated PTX.

In a recent study of breast cancer patients published by Lodola et al, a finding suggested that the intracellular $\mathrm{Ca}^{2+}$ toolkit, which is responsible for the pro-angiogenic effect of VEGF, is remodeled in cancer patients and rendered insensitive to VEGF (17). This suggests that these tumor cells are resistant to the angiogenic effect of VEGF, and may consequently be resistant to VEGF inhibitors as anti-angiogenic agents. This finding suggests that VEGF inhibitors (including $\mathrm{BV}$ ), may not have as important a role in tumor vascularization as previously thought, and raises the possibility that PTX, in addition to the other adverse effects of these agents, may be a result of an off-target effect rather than an anti-angiogenic effect. Further in vitro studies that investigate this off-target effect are required. These studies may also uncover, as the abovementioned study, potential novel targets, such as the store-operated $\mathrm{Ca}^{2+}$ entry mechanism.

It is difficult to ascertain the exact frequency of BV-associated PTX, given the scarcity of reported cases, and 
the inability to ascertain the number of patients receiving BV therapy annually. However, Interiano et al conducted a retrospective analysis of selected pediatric patients with recurrent or refractory solid malignancies who had undergone combination therapy with BV and sorafenib with low-dose cyclophosphamide therapy. The goal of the analysis was to assess the risk of developing PTX. The study reported an unexpectedly high incidence of PTX in 11 of the 44 subjects (25\%) (18). Although that study was conducted in pediatric patients, its results are significant and suggest that BV-associated PTX may be occurring at higher rates than reported as compared to healthy individuals. In comparison, primary spontaneous PTX (PSP) in healthy individuals is estimated to occur at a rate of 7.4-18 cases per 100,000 amongst males, and 6 cases per 100,000 amongst females. In addition, PSP rarely occurs after the age of 40 years (19).

It is possible that the lack of a significant number of case reports describing PTX in association with BV therapy is due to the lack of widespread knowledge of this association; hence, there may be a number of unreported such cases. Therefore, it is important to report these cases to spread awareness amongst clinicians to this potentially life-threatening collateral effect of BV. There is also the need for further studies to establish a causal association between BV and PTX and elucidate the mechanisms underlying this effect.

\section{Acknowledgements}

The authors would like to thank Dr Asem Al-Refaie for his comments on the manuscript.

\section{Funding}

No funding was received.

\section{Availability of data and materials}

All data generated/analyzed in the present study are included in the published manuscript.

\section{Authors' contributions}

TA contributed to the conception of the work. TA, RS, DR, KK and SP contributed to the drafting of the manuscript, critical review of the article, and approval of the final version. All authors have read and approved the final version of the manuscript.

\section{Ethics approval and consent to participate}

Not applicable.

\section{Patient consent to publication}

Verbal informed consent was obtained from the patient for the publication of this case' details and associated images.

\section{Competing interests}

The authors declare that they have no competing interests.

\section{References}

1. Shibuya M: Vascular endothelial growth factor (VEGF) and its receptor (VEGFR) signaling in angiogenesis: A crucial target for anti- and pro-angiogenic therapies. Genes Cancer 2: 1097-1105, 2011.

2. Hurwitz H, Fehrenbacher L, Novotny W, Cartwright T, Hainsworth J, Heim W, Berlin J, Baron A, Griffing S, Holmgren E, et al: Bevacizumab plus irinotecan, fluorouracil, and leucovorin for metastatic colorectal cancer. N Engl J Med 350: 2335-2342, 2004.

3. Ilic I, Jankovic S and Ilic M: Bevacizumab combined with chemotherapy improves survival for patients with metastatic colorectal cancer: Evidence from meta analysis. PLoS One 11: e0161912, 2016.

4. Keating GM: Bevacizumab: A review of its use in advanced cancer. Drugs 74: 1891-1925, 2014.

5. Totzeck M, Mincu RI and Rassaf T: Cardiovascular adverse events in patients with cancer treated with bevacizumab: A meta-analysis of more than 20000 patients. J Am Heart Assoc 6: e006278, 2017.

6. Zhang Y, Yang H, Zhao M and He J: Bilateral pneumothorax after bevacizumab-containing chemotherapy in fibrosarcoma. J Thorac Dis 4: 229-231, 2012.

7. Yang SH, Lin JK, Chen WS, Lin TC, Yang SH, Jiang JK, Chang SC, Lan YT, Chao TC, Yen CC, et al: Pneumothorax after bevacizumab-containing chemotherapy: A case report. Jpn J Clin Oncol 41: 269-271, 2011.

8. Makino T, Kudo S and Ogata T: Pneumothorax after treatment with bevacizumab-containing chemotherapy for breast cancer-a case report. Gan To Kagaku Ryoho 41: 233-235, 2014 (In Japanese)

9. Koh H, Kamiishi N, Kimura Y, Tajima A, Yagami T and Mukai M: A rare case of persistent pneumothorax in non-small cell lung cancer on bevacizumab therapy. J Pulm Respir Med: Mar 26, 2013 (Epub ahead of print). doi: 10.4172/2161-105X. S14-001.

10. Iida T, Yabana T, Nakagaki S, Adachi T and Kondo Y: A: rupture of a lung metastatic lesion of colon cancer, leading to pneumothorax caused by bevacizumab. Intern Med 55: 3125-3129, 2016.

11. Bazan F, Vollmer I and Gayete A: Chemotherapy-induced secondary pneumothorax. Arch Bronconeumol 50: 44, 2014.

12. Ueda Y, Huang CL, Itotani R and Fukui M: Endobronchial watanabe spigot placement for a secondary pneumothorax. J Bronchology Interv Pulmonol 22: 278-280, 2015.

13. Qiu M, Hu J, Yang D, Cosgrove DP and Xu R: Pattern of distant metastases in colorectal cancer: A SEER based study. Oncotarget 6: 38658-38666, 2015.

14. Srinivas S and Varadhachary G: Spontaneous pneumothorax in malignancy: A case report and review of the literature. Ann Oncol 11: 887-889, 2000.

15. Kasahara Y, Tuder RM, Taraseviciene-Stewart L, Le Cras TD, Abman S, Hirth PK, Waltenberger J and Voelkel NF: Inhibition of VEGF receptors causes lung cell apoptosis and emphysema. J Clin Invest 106: 1311-1319, 2000.

16. Collins BT, Vahdat S, Erickson K, Collins SP, Suy S, Yu X, Zhang Y, Subramaniam D, Reichner CA, Sarikaya I, et al: Radical cyberknife radiosurgery with tumor tracking: An effective treatment for inoperable small peripheral stage I non-small cell lung cancer. J Hematol Oncol 2: 1, 2009.

17. Lodola F, Laforenza U, Cattaneo F, Ruffinatti FA, Poletto V, Massa M, Tancredi R, Zuccolo E, Khdar DA, Riccardi A, et al: VEGF-induced intracellular $\mathrm{Ca}^{2+}$ oscillations are down-regulated and do not stimulate angiogenesis in breast cancer-derived endothelial colony forming cells. Oncotarget 8: 95223-95246, 2017.

18. Interiano RB, McCarville MB, Wu J, Davidoff AM, Sandoval J and Navid F: Pneumothorax as a complication of combination antiangiogenic therapy in children and young adults with refractory/recurrent solid tumors. J Pediatr Surg 50: 1484-1489, 2015.

19. Sahn SA and Heffner JE: Spontaneous pneumothorax. N Engl J Med 342: 868-874, 2000. 